\title{
Symmetries of the Maxwell-Bloch equations with the rotating wave approximation
}

\author{
Ioan Caşu \\ Departamentul de Matematică, Universitatea de Vest din Timişoara \\ Bd. V. Pârvan, Nr. 4, 300223 Timişoara, România \\ E-mail: casu@math.uvt.ro
}

\begin{abstract}
In this paper a symplectic realization for the Maxwell-Bloch equations with the rotating wave approximation is given, which also leads to a Lagrangian formulation. We show how Lie point symmetries generate a third constant of motion for the considered dynamical system.
\end{abstract}

Keywords: Maxwell-Bloch equations, symmetries, Hamiltonian dynamics, Lagrangian dynamics.

\section{Introduction}

The Maxwell-Bloch laser equations have significant importance in optics where they describe the interaction between laser light and a material sample composed of two-level atoms. A nice presentation of the Maxwell-Bloch dynamics is given by David and Holm in David \& Holm (1992)]. Besides its physical interest, the 3-dimensional real valued Maxwell-Bloch equations have been widely investigated from the different points of view: homoclinic chaos [Holm et al. (1991)], Lie-Poisson Hamiltonian structures David \& Holm (1992)], integrability and geometric prequantization Puta (1998)], symmetries Damianou \& Paschali (1995)], periodic orbits and energy-Casimir map Lăzureanu et al. (2010)]. Considering a control, Puta [Puta (1996)] studied the stability problem and Bînzar \& Lăzureanu [Bînzar \& Lăzureanu (2012), Bînzar \& Lăzureanu (2013)] studied some properties of energy-Casimir map.

The present work deals with five dimensional real valued Maxwell-Bloch equations with the rotating wave approximation,

$$
\left\{\begin{array}{l}
\dot{x}_{1}=y_{1} \\
\dot{y}_{1}=x_{1} z \\
\dot{x}_{2}=y_{2} \\
\dot{y}_{2}=x_{2} z \\
\dot{z}=-\left(x_{1} y_{1}+x_{2} y_{2}\right) .
\end{array}\right.
$$

This system has been recently studied by Huang [Huang (2004)] (bi-Hamiltonian structure, homoclinic orbits) and Birtea \& Caşu [Birtea \& Caşu (2013)] (stability of equilibria, homoclinic and periodic orbits).

In the following some symmetries of system (1.1) are considered. The importance of knowing the symmetry group is reflected by using it to determine some special types of solutions.

The symmetry approach for even order systems of differential equations can be found in Leach (1981), Damianou (1990), Damianou \& Sophocleus (1999), Damianou \& Sophocleus (2000).

For a class of three dimensional dynamical systems different types of symmetries have been computed in Lăzureanu \& Bînzar (2012), Bînzar \& Lăzureanu (2012), Lăzureanu \& Bînzar (2013)]

Theoretical details about symmetries of differential equations can be found in [Fuchssteiner (1983)], [Fokas \& Fuchssteiner (1981], Olver (1986)], Bluman \& Kumei (1989)], Damianou (2000)].

The second section of the present paper studies the Hamiltonian structure of the considered system and its Lagrangian representation. Thus, a Lie group is determined and its associated Lie algebra defines 
a Poisson structure. Moreover, a symplectic realization and a Lagrangian realization of system (1.1) are given.

In section three, the Lie point symmetries of the Euler-Lagrange equations are studied. These symmetries form a four dimensional Lie algebra and some of them are variational symmetries related with the constants of motion of our system. Also, Lie point symmetries and master symmetries are found.

\section{Hamiltonian structures}

In this section a Poisson structure of system (1.1) is constructed and a symplectic realization of the system (1.1) is given.

Recall that for system (1.1), the functions $H, C \in \mathcal{C}^{\infty}\left(\mathbb{R}^{5}, \mathbb{R}\right)$

$$
H\left(x_{1}, y_{1}, x_{2}, y_{2}, z\right)=\frac{1}{2}\left(y_{1}^{2}+y_{2}^{2}+z^{2}\right),
$$

and

$$
C\left(x_{1}, y_{1}, x_{2}, y_{2}, z\right)=\frac{1}{2}\left(x_{1}^{2}+x_{2}^{2}\right)+z
$$

are constants of motion.

In order to obtain a Poisson structure, let us consider the linear Poisson bracket $\{\cdot, \cdot\}$,

$$
\left\{u_{i}, u_{j}\right\}=\sum_{k=1}^{5} \alpha_{i j}^{k} u_{k}+\beta_{i j}, i<j,
$$

where $u_{1}=x_{1}, u_{2}=y_{1}, u_{3}=x_{2}, u_{4}=y_{2}, u_{5}=z$ and $\alpha_{i j}^{k}, \beta_{i j} \in \mathbb{R}$.

Imposing the condition that $C$ is a Casimir for (2.1) and $H$ is a Hamiltonian function, we get a dynamical system which coincides with the system (1.1) only if

$$
\left\{u_{1}, u_{2}\right\}=1,\left\{u_{2}, u_{5}\right\}=u_{1},\left\{u_{3}, u_{4}\right\}=1,\left\{u_{4}, u_{5}\right\}=u_{3}
$$

and $\left\{u_{i}, u_{j}\right\}=0$ otherwise.

Therefore we consider the five-dimensional Lie algebra given by

$$
\left[E_{2}, E_{5}\right]=E_{1},\left[E_{4}, E_{5}\right]=E_{3}
$$

where

$$
\begin{gathered}
E_{1}=\left[\begin{array}{cccc}
0 & 0 & -1 & 0 \\
0 & 0 & 0 & 0 \\
0 & 0 & 0 & 0 \\
0 & 0 & 0 & 0
\end{array}\right] ; \quad E_{2}=\left[\begin{array}{cccc}
0 & 0 & 0 & 0 \\
0 & 0 & -1 & 0 \\
0 & 0 & 0 & 0 \\
0 & 0 & 0 & 0
\end{array}\right] ; \quad E_{3}=\left[\begin{array}{cccc}
0 & 0 & 0 & 1 \\
0 & 0 & 0 & 0 \\
0 & 0 & 0 & 0 \\
0 & 0 & 0 & 0
\end{array}\right] ; \\
E_{4}=\left[\begin{array}{cccc}
0 & 0 & 0 & 0 \\
0 & 0 & 0 & 1 \\
0 & 0 & 0 & 0 \\
0 & 0 & 0 & 0
\end{array}\right] ; E_{5}=\left[\begin{array}{cccc}
0 & -1 & 0 & 0 \\
0 & 0 & 0 & 0 \\
0 & 0 & 0 & 0 \\
0 & 0 & 0 & 0
\end{array}\right] .
\end{gathered}
$$

The Lie algebra generated by the base $B=\left\{E_{1}, E_{2}, E_{3}, E_{4}, E_{5}\right\}$ is the subalgebra

$$
\mathfrak{g}=n_{5}^{5}=\left\{E=\left[\begin{array}{cccc}
0 & -\theta & -\alpha & \gamma \\
0 & 0 & -\beta & \delta \\
0 & 0 & 0 & 0 \\
0 & 0 & 0 & 0
\end{array}\right] \mid \alpha, \beta, \gamma, \delta, \theta \in \mathbb{R}\right\}
$$


of the Lie algebra $g_{4}$, see Benjumea et al. (2006), and the corresponding Lie group is given by

$$
N_{5}^{5}=\left\{X=\left[\begin{array}{cccc}
1 & -r & -m & p \\
0 & 1 & -n & q \\
0 & 0 & 1 & 0 \\
0 & 0 & 0 & 1
\end{array}\right] \mid m, n, p, q \in \mathbb{R}\right\}
$$

We observe that the Lie algebras $(\mathfrak{g},+, \cdot,[\cdot, \cdot])$ and $\left(\mathbb{R}^{5},+, \cdot, \times\right)$ are isomorphic, where the product $\times: \mathbb{R}^{5} \times \mathbb{R}^{5} \rightarrow \mathbb{R}^{5}$ is defined by

$$
\left(\alpha_{1}, \beta_{1}, \gamma_{1}, \delta_{1}, \theta_{1}\right) \times\left(\alpha_{2}, \beta_{2}, \gamma_{2}, \delta_{2}, \theta_{2}\right)=\left(\beta_{1} \theta_{2}-\beta_{2} \theta_{1}, 0, \delta_{1} \theta_{2}-\delta_{2} \theta_{1}, 0,0\right) .
$$

Indeed, an easy computation shows that the map

$$
\Phi:(\alpha, \beta, \gamma, \delta, \theta) \in \mathbb{R}^{5} \mapsto\left[\begin{array}{cccc}
0 & -\theta & -\alpha & \gamma \\
0 & 0 & -\beta & \delta \\
0 & 0 & 0 & 0 \\
0 & 0 & 0 & 0
\end{array}\right] \in \mathfrak{g}
$$

is a Lie algebra isomorphism.

Let us consider the bilinear map $\Theta: \mathfrak{g} \times \mathfrak{g} \rightarrow \mathbb{R}$ given by the matrix

$$
\Theta=\left[\begin{array}{ccccc}
0 & 1 & 0 & 0 & 0 \\
-1 & 0 & 0 & 0 & 0 \\
0 & 0 & 0 & 1 & 0 \\
0 & 0 & -1 & 0 & 0 \\
0 & 0 & 0 & 0 & 0
\end{array}\right]
$$

Following Libermann \& Marle (1987)], $\Theta$ is a 2-cocycle on $\mathfrak{g}$ and it is not a coboundary since $\Theta\left(E_{1}, E_{2}\right)=$ $1 \neq 0=f\left(\left[E_{1}, E_{2}\right]\right)$, for every linear map $f, f: \mathfrak{g} \rightarrow \mathbb{R}$.

Therefore, on the dual space $\mathfrak{g}^{*} \simeq \mathbb{R}^{5}$, a modified Lie-Poisson structure is given in coordinates by

$$
\pi=\left[\begin{array}{ccccc}
0 & 0 & 0 & 0 & 0 \\
0 & 0 & 0 & 0 & x_{1} \\
0 & 0 & 0 & 0 & 0 \\
0 & 0 & 0 & 0 & x_{2} \\
0 & -x_{1} & 0 & -x_{2} & 0
\end{array}\right]+\left[\begin{array}{ccccc}
0 & 1 & 0 & 0 & 0 \\
-1 & 0 & 0 & 0 & 0 \\
0 & 0 & 0 & 1 & 0 \\
0 & 0 & -1 & 0 & 0 \\
0 & 0 & 0 & 0 & 0
\end{array}\right]=\left[\begin{array}{ccccc}
0 & 1 & 0 & 0 & 0 \\
-1 & 0 & 0 & 0 & x_{1} \\
0 & 0 & 0 & 1 & 0 \\
0 & 0 & -1 & 0 & x_{2} \\
0 & -x_{1} & 0 & -x_{2} & 0
\end{array}\right]
$$

Hence $\left(\mathbb{R}^{5}, \pi, X_{H}\right)$ is a Hamilton-Poisson realization of the dynamics (1.1), where $X_{H}=\left(y_{1}, x_{1} z, y_{2}, x_{2} z\right.$, $\left.-x_{1} y_{1}-x_{2} y_{2}\right)$.

The next theorem states that the system (1.1) has a symplectic realization.

Theorem 2.1. The Hamilton-Poisson mechanical system $\left(\mathbb{R}^{5}, \pi, X_{H}\right)$ has a full symplectic realization $\left(T^{*} \mathbb{R}^{3} \simeq \mathbb{R}^{6}, \omega, X_{\tilde{H}}\right)$, with the canonical symplectic form

$$
\omega=d p_{1} \wedge d q_{1}+d p_{2} \wedge d q_{2}+d p_{3} \wedge d q_{3}
$$

the Hamiltonian

$$
\tilde{H}=\frac{1}{2} p_{1}^{2}+\frac{1}{2} p_{2}^{2}+\frac{1}{2}\left[p_{3}-\frac{1}{2}\left(q_{1}^{2}+q_{2}^{2}\right)\right]^{2}
$$

and the corresponding Hamiltonian vector field is given by

$X_{\tilde{H}}=p_{1} \frac{\partial}{\partial q_{1}}+p_{2} \frac{\partial}{\partial q_{2}}+\left[p_{3}-\frac{1}{2}\left(q_{1}^{2}+q_{2}^{2}\right)\right] \frac{\partial}{\partial q_{3}}+\left[q_{1} p_{3}-\frac{1}{2} q_{1}^{3}-\frac{1}{2} q_{1} q_{2}^{2}\right] \frac{\partial}{\partial p_{1}}+\left[q_{2} p_{3}-\frac{1}{2} q_{1}^{2} q_{2}-\frac{1}{2} q_{2}^{3}\right] \frac{\partial}{\partial p_{2}}$. 
Proof. For the Hamiltonian $\tilde{H}$ the corresponding Hamilton's equations are

$$
\left\{\begin{array}{l}
\dot{q}_{1}=p_{1} \\
\dot{q}_{2}=p_{2} \\
\dot{q}_{3}=p_{3}-\frac{1}{2}\left(q_{1}^{2}+q_{2}^{2}\right) \\
\dot{p}_{1}=q_{1} p_{3}-\frac{1}{2} q_{1}^{3}-\frac{1}{2} q_{1} q_{2}^{2} \\
\dot{p}_{2}=q_{2} p_{3}-\frac{1}{2} q_{1}^{2} q_{2}-\frac{1}{2} q_{2}^{3} \\
\dot{p}_{3}=0 .
\end{array}\right.
$$

We define the application

$$
\varphi: \mathbb{R}^{6} \rightarrow \mathbb{R}^{5}, \varphi\left(q_{1}, q_{2}, q_{3}, p_{1}, p_{2}, p_{3}\right)=\left(x_{1}, y_{1}, x_{2}, y_{2}, z\right),
$$

where

$$
x_{1}=q_{1}, y_{1}=p_{1}, x_{2}=q_{2}, y_{2}=p_{2}, z=p_{3}-\frac{1}{2}\left(q_{1}^{2}+q_{2}^{2}\right) .
$$

It follows that $\varphi$ is a surjective submersion, the Hamiltonian vector field $X_{\tilde{H}}$ is mapped onto the Hamiltonian vector field $X_{H}$ (the equations (2.2) are mapped onto the equations (1.1)), the canonical structure $\{., .\}_{\omega}$ is mapped onto the Poisson structure $\pi$, as required.

We remark that $H \circ \varphi=\tilde{H}$.

We also denote $\tilde{C}:=C \circ \varphi=p_{3}$.

The following result shows that system (2.2) can be written in Lagrangian formalism.

Theorem 2.2. The system (2.2) takes the form

$$
\left\{\begin{array}{l}
\ddot{q}_{1}-q_{1} \dot{q}_{3}=0 \\
\ddot{q}_{2}-q_{2} \dot{q}_{3}=0 \\
\ddot{q}_{3}+q_{1} \dot{q}_{1}+q_{2} \dot{q}_{2}=0
\end{array}\right.
$$

on the tangent bundle $T \mathbb{R}^{3}$.

Moreover, the system (2.3) represents the Euler-Lagrange equations generated by the Lagrangian

$$
L=\frac{1}{2} \dot{q}_{1}^{2}+\frac{1}{2} \dot{q}_{2}^{2}+\frac{1}{2} \dot{q}_{3}^{2}+\frac{1}{2} \dot{q}_{3}\left(q_{1}^{2}+q_{2}^{2}\right) .
$$

Proof. From Hamilton's equations (2.2) we obtain by differentiation equations (2.3). Also, for the Lagrangian $L$, the Euler-Lagrange equations $\frac{d}{d t} \frac{\partial L}{\partial \dot{q}_{i}}-\frac{\partial L}{\partial q_{i}}=0$ takes the form (2.3).

Using Legendre transform $\mathbb{F} L: T \mathbb{R}^{3} \rightarrow T^{*} \mathbb{R}^{3}, \mathbb{F} L\left(q_{1}, q_{2}, q_{3}, \dot{q}_{1}, \dot{q}_{2}, \dot{q}_{3}\right)=\left(q_{1}, q_{2}, q_{3}, p_{1}, p_{2}, p_{3}\right)$, where $p_{i}=\frac{\partial L}{\partial \dot{q}_{i}}$, the relation between the Hamiltonian $\tilde{H}$ and the Lagrangian $L, \tilde{H}=\sum p_{i} \dot{q}_{i}-L$, holds.

For details about Lagrangian and Hamiltonian formalism see, for example, Marsden \& Raţiu (1999)].

\section{Symmetries}

In this section several types of symmetries are studied. In the beginning, the Lie point symmetries of system (2.3) are computed. Then these symmetries are transformed in Lie point symmetries, respectively symmetries and master symmetries for system (1.1).

A vector field

$$
\mathbf{u}=\xi\left(q_{1}, q_{2}, q_{3}, t\right) \frac{\partial}{\partial t}+\eta_{1}\left(q_{1}, q_{2}, q_{3}, t\right) \frac{\partial}{\partial q_{1}}+\eta_{2}\left(q_{1}, q_{2}, q_{3}, t\right) \frac{\partial}{\partial q_{2}}+\eta_{3}\left(q_{1}, q_{2}, q_{3}, t\right) \frac{\partial}{\partial q_{3}}
$$


is a Lie-point symmetry for Euler-Lagrange equations (2.3) if the action of its second prolongation on these equations vanishes, where

$$
p r^{(2)}(\mathbf{u})=\mathbf{u}+\sum\left(\dot{\eta}_{i}-\dot{\xi} \dot{q}_{i}\right) \frac{\partial}{\partial \dot{q}_{i}}+\sum\left(\ddot{\eta}_{i}-\ddot{\xi}_{\dot{q}_{i}}-2 \dot{\xi} \ddot{q}_{i}\right) \frac{\partial}{\partial \ddot{q}_{i}} .
$$

Thus the following relations are obtained:

$$
\begin{aligned}
& \ddot{\eta}_{1}-\ddot{\xi}_{\dot{q}_{1}}-2 \ddot{q}_{1} \dot{\xi}-\eta_{1} \dot{q}_{3}-q_{1}\left(\dot{\eta}_{3}-\dot{\xi} \dot{q}_{3}\right)=0 \\
& \ddot{\eta}_{2}-\ddot{\xi} \dot{q}_{2}-2 \ddot{q}_{2} \dot{\xi}-\eta_{2} \dot{q}_{3}-q_{2}\left(\dot{\eta}_{3}-\dot{\xi} \dot{q}_{3}\right)=0 \\
& \ddot{\eta}_{3}-\ddot{\xi} \dot{q}_{3}-2 \ddot{q}_{3} \dot{\xi}+\eta_{1} \dot{q}_{1}+\eta_{2} \dot{q}_{2}+q_{1}\left(\dot{\eta}_{1}-\dot{\xi} \dot{q}_{1}\right)+q_{2}\left(\dot{\eta}_{2}-\dot{\xi} \dot{q}_{2}\right)=0 .
\end{aligned}
$$

The resulting equations obtained by expanding $\dot{\xi}, \ddot{\xi}, \dot{\eta}_{1}, \ddot{\eta}_{1}, \dot{\eta}_{2}, \ddot{\eta}_{2}, \dot{\eta}_{3}, \ddot{\eta}_{3}$ and replacing $\ddot{q}_{1}, \ddot{q}_{2}$ and $\ddot{q}_{3}$ must be satisfied identically in $t, q_{1}, q_{2}, q_{3}, \dot{q}_{1}, \dot{q}_{2}, \dot{q}_{3}$, which are all independent variables. By performing straightforward computations, we get the overall result:

$$
\xi=-\alpha t+\beta, \quad \eta_{1}=\alpha q_{1}+\gamma q_{2}, \quad \eta_{2}=-\gamma q_{1}+\alpha q_{2}, \quad \eta_{3}=\alpha q_{3}+\delta,
$$

where $\alpha, \beta, \gamma, \delta$ are real constants.

We can summarize the above considerations in the following result.

Theorem 3.1. The symmetries of equations (2.3) are given by

$$
\mathbf{u}=(-\alpha t+\beta) \frac{\partial}{\partial t}+\left(\alpha q_{1}+\gamma q_{2}\right) \frac{\partial}{\partial q_{1}}+\left(-\gamma q_{1}+\alpha q_{2}\right) \frac{\partial}{\partial q_{2}}+\left(\alpha q_{3}+\delta\right) \frac{\partial}{\partial q_{3}},
$$

where $\alpha, \beta, \gamma, \delta \in \mathbb{R}$.

The next proposition provides the algebraic structure of the above symmetries.

Proposition 3.2. The symmetries of equations (2.3) form a 4-dimensional Lie algebra $\mathfrak{s}$.

Proof. This Lie algebra is generated by the base $\left\{\mathbf{u}_{1}, \mathbf{u}_{2}, \mathbf{u}_{3}, \mathbf{u}_{4}\right\}$, where

$$
\begin{aligned}
& \mathbf{u}_{1}=-t \frac{\partial}{\partial t}+q_{1} \frac{\partial}{\partial q_{1}}+q_{2} \frac{\partial}{\partial q_{2}}+q_{3} \frac{\partial}{\partial q_{3}} \\
& \mathbf{u}_{2}=\frac{\partial}{\partial t} \\
& \mathbf{u}_{3}=\frac{\partial}{\partial q_{3}} \\
& \mathbf{u}_{4}=q_{2} \frac{\partial}{\partial q_{1}}-q_{1} \frac{\partial}{\partial q_{2}}
\end{aligned}
$$

and the Lie algebra bracket is given by:

$$
\left[\mathbf{u}_{1}, \mathbf{u}_{2}\right]=\mathbf{u}_{2}, \quad\left[\mathbf{u}_{1}, \mathbf{u}_{3}\right]=-\mathbf{u}_{3}, \quad\left[\mathbf{u}_{1}, \mathbf{u}_{4}\right]=0, \quad\left[\mathbf{u}_{2}, \mathbf{u}_{3}\right]=0, \quad\left[\mathbf{u}_{2}, \mathbf{u}_{4}\right]=0, \quad\left[\mathbf{u}_{3}, \mathbf{u}_{4}\right]=0 .
$$

Now, we consider the matrix Lie algebra generated by the base $B=\left\{A_{1}, A_{2}, A_{3}, A_{4}\right\}$,

$$
\begin{gathered}
A_{1}=\left[\begin{array}{cccc}
0 & 0 & 0 & 0 \\
0 & 0 & 0 & 0 \\
0 & 0 & -1 & 0 \\
0 & 0 & 0 & 1
\end{array}\right] ; A_{2}=\left[\begin{array}{cccc}
0 & 0 & 1 & 0 \\
0 & 0 & 0 & 0 \\
0 & 0 & 0 & 0 \\
0 & 0 & 0 & 0
\end{array}\right] ; \\
A_{3}=\left[\begin{array}{cccc}
0 & 0 & 0 & 1 \\
0 & 0 & 0 & 0 \\
0 & 0 & 0 & 0 \\
0 & 0 & 0 & 0
\end{array}\right] ; A_{4}=\left[\begin{array}{cccc}
0 & 1 & 0 & 0 \\
0 & 0 & 0 & 0 \\
0 & 0 & 0 & 0 \\
0 & 0 & 0 & 0
\end{array}\right],
\end{gathered}
$$


namely

$$
\mathfrak{s}_{\mathfrak{g}}=\left\{A=\left[\begin{array}{cccc}
0 & d & b & c \\
0 & 0 & 0 & 0 \\
0 & 0 & -a & 0 \\
0 & 0 & 0 & a
\end{array}\right] \mid a, b, c, d \in \mathbb{R}\right\} .
$$

The following relations

$$
\left[A_{1}, A_{2}\right]=A_{2},\left[A_{1}, A_{3}\right]=-A_{3},\left[A_{1}, A_{4}\right]=0,\left[A_{2}, A_{3}\right]=0,\left[A_{2}, A_{4}\right]=0,\left[A_{3}, A_{4}\right]=0
$$

hold. Hence the Lie algebras $\mathfrak{s}$ and $\mathfrak{s}_{\mathfrak{g}}$ are isomorphic.

The Lie group corresponding to Lie algebra $\mathfrak{s}_{\mathfrak{g}}$ is given by

$$
S_{G}=\left\{X=\left[\begin{array}{cccc}
1 & w & u & v \\
0 & 1 & 0 & 0 \\
0 & 0 & e^{-s} & 0 \\
0 & 0 & 0 & e^{s}
\end{array}\right] \mid s, u, v, w \in \mathbb{R}\right\}
$$

The following proposition furnishes variational symmetries of Euler-Lagrange equations (2.3).

Proposition 3.3. In the case $\alpha=0$ the Lie point symmetries $\mathbf{u}$ given by (3.1) are variational symmetries of equations (2.3).

Proof. The vector field $\mathbf{u}$ with the infinitesimal generators $\xi, \eta_{1}, \eta_{2}, \eta_{3}$ is a variational symmetry if and only if $\operatorname{pr}^{(1)}(\mathbf{u}) L+L \dot{\xi}=0$, see Brunt (2004)]. In our case

$$
\begin{aligned}
p r^{(1)}(\mathbf{u}) & =(-\alpha t+\beta) \frac{\partial}{\partial t}+\left(\alpha q_{1}+\gamma q_{2}\right) \frac{\partial}{\partial q_{1}}+\left(-\gamma q_{1}+\alpha q_{2}\right) \frac{\partial}{\partial q_{2}}+\left(\alpha q_{3}+\delta\right) \frac{\partial}{\partial q_{3}} \\
& +\left(2 \alpha \dot{q}_{1}+\gamma \dot{q}_{2}\right) \frac{\partial}{\partial \dot{q}_{1}}+\left(2 \alpha \dot{q}_{2}-\gamma \dot{q}_{1}\right) \frac{\partial}{\partial \dot{q}_{2}}+2 \alpha \dot{q}_{3} \frac{\partial}{\partial \dot{q}_{3}} .
\end{aligned}
$$

Therefore $\operatorname{pr}^{(1)}(\mathbf{u}) L+L \dot{\xi}=3 \alpha L$ and the conclusion follows.

Remark 3.1. It is known that variational symmetries give rise to constants of motion. More precisely, using Noether's theorem ([Brunt (2004), Noether (1918)] $)$ we obtain that

$$
I=-\beta \tilde{H}-\gamma \tilde{J}+\delta \tilde{C}
$$

is constant of motion for equations (2.3), where $\tilde{J}=q_{1} \dot{q}_{2}-q_{2} \dot{q}_{1}$, or, using Legendre transformation, $\tilde{J}=q_{1} p_{2}-q_{2} p_{1}$. Moreover, $\mathbf{u}_{2}$ represents the time translation symmetry which generates the conservation of energy $H, \mathbf{u}_{3}$ represents a translation in the cyclic $q_{3}$ direction which is related to the conservation of the conjugate momentum $p_{3}=\tilde{C}$. Also, the variational symmetry $\mathbf{u}_{4}$ represents a rotation around $q_{3}$ axis and the corresponding constant of motion $\tilde{J}=q_{1} p_{2}-q_{2} p_{1}$ is the third component of the angular momentum vector $\mathbf{q} \times \mathbf{p}$.

Theorem 3.4. Solving $\tilde{J}=J \circ \varphi$ it follows that $J=x_{1} y_{2}-x_{2} y_{1}$ is a third constant of motion for the system (1.1).

Taking into account the relationship between Maxwell-Bloch equation (1.1) and Euler-Lagrange equations (2.3) it is natural to ask what connections are between the symmetries of these systems.

Using the push forward on a vector field by $\mathbb{F} L$, one gets the corresponding vector field on $T^{*} \mathbb{R}^{3}$ :

$$
\begin{aligned}
(\mathbb{F} L)_{*}\left(p r^{(1)}(\mathbf{u})\right) & =(-\alpha t+\beta) \frac{\partial}{\partial t}+\left(\alpha q_{1}+\gamma q_{2}\right) \frac{\partial}{\partial q_{1}}+\left(-\gamma q_{1}+\alpha q_{2}\right) \frac{\partial}{\partial q_{2}}+\left(\alpha q_{3}+\delta\right) \frac{\partial}{\partial q_{3}} \\
& +\left(2 \alpha p_{1}+\gamma p_{2}\right) \frac{\partial}{\partial p_{1}}+\left(2 \alpha p_{2}-\gamma p_{1}\right) \frac{\partial}{\partial p_{2}}+2 \alpha p_{3} \frac{\partial}{\partial p_{3}}
\end{aligned}
$$


denoted by $\tilde{\mathbf{v}}$. Applying the push forward on the vector field $\tilde{\mathbf{v}}$ by $\varphi$ one obtains the following vector field:

$$
\begin{aligned}
\mathbf{X} & =(-\alpha t+\beta) \frac{\partial}{\partial t}+\left(\alpha x_{1}+\gamma x_{2}\right) \frac{\partial}{\partial x_{1}}+\left(2 \alpha y_{1}+\gamma y_{2}\right) \frac{\partial}{\partial y_{1}}+\left(-\gamma x_{1}+\alpha x_{2}\right) \frac{\partial}{\partial x_{2}} \\
& +\left(2 \alpha y_{2}-\gamma y_{1}\right) \frac{\partial}{\partial y_{2}}+2 \alpha z \frac{\partial}{\partial z} .
\end{aligned}
$$

Now, we can present symmetries of Maxwell-Bloch equations (1.1).

Proposition 3.5. (i) The vector field (3.2) is a Lie point symmetry of (1.1). Moreover, it is a conformal symmetry and also a master symmetry.

(ii) In the case $\alpha=0$, the vector field

$$
\mathbf{X}=\beta \frac{\partial}{\partial t}+\gamma x_{2} \frac{\partial}{\partial x_{1}}+\gamma y_{2} \frac{\partial}{\partial y_{1}}-\gamma x_{1} \frac{\partial}{\partial x_{2}}-\gamma y_{1} \frac{\partial}{\partial y_{2}},
$$

is a symmetry of (1.1).

Acknowledgements. This work was supported by a grant of the Romanian National Authority for Scientific Research, CNCS - UEFISCDI, project number PN-II-RU-TE-2011-3-0006.

\section{References}

[Benjumea et al. (2006)] J. C. Benjumea, F. J. Echarte, J. Nunez, A. F. Tenorio, Simply connected Lie subgroups of the unipotent Lie group of order 4, Soochow J. of Math., Volume 32, No. 2 (2006) $181-199$.

[Birtea \& Caşu (2013)] P. Birtea, I. Caşu, The stability problem and special solutions for the 5components MaxwellBloch equations, Applied Mathematics Letters Volume 26, Issue 8 (2013) $875-880$.

[Bînzar \& Lăzureanu (2012)] C. Lăzureanu, T. Bînzar, Some geometrical properties of the MaxwellBloch equations with a linear control, Proc. of the XIII-th Int. Conf. on Mathematics and its Applications, Timişoara, 2012, 151-158.

[Bînzar \& Lăzureanu (2012)] T. Bînzar, C. Lăzureanu, On the symmetries of a Rabinovich type system, Scientific Bulletin of the "Politehnica" University of Timişoara, Transactions on Mathenatics \& Physics, Tom 57(71), Fascicola 2 (2012) 29-36.

[Bînzar \& Lăzureanu (2013)] T. Bînzar, C. Lăzureanu, On some dynamical and geometrical properties of the Maxwell-Bloch equations with a quadratic control, Journal Of Geometry and Physics, Vol. 70 (2013) 1-8.

[Bluman \& Kumei (1989)] G.W. Bluman, S. Kumei, Symmetries and Differential Equations, Appl. Math. Sci., No. 81, Springer Verlag, New York, 1989.

[Brunt (2004)] B. van Brunt, The calculus of variations, Springer Verlag, New York, 2004.

[Damianou (1990)] P. Damianou, Master symmetries and R-matrices for the Toda Lattice, Lett. in Math. Phys. 20 (1990) 101-112.

[Damianou (2000)] P.A. Damianou, Multiple hamiltonian structures for Toda systems of type A-B-C, Regular and Chaotic Dynamics, V. 5, No. 1 (2000) 17-32.

[Damianou \& Paschali (1995)] P.A. Damianou, P.G. Paschali, Symmetries of Maxwell-Bloch equations, J.Nonlinear Math.Phys., 2 N 3-4 (1995) 269-278. 
[Damianou \& Sophocleus (1999)] P. A. Damianou, C. Sophocleous, Symmetries of Hamiltonian systems with two degrees of freedom, J. Math. Phys. 40 (1999) 210.

[Damianou \& Sophocleus (2000)] P. A. Damianou, C. Sophocleous, Symmetry group classification of three-dimensional Hamiltonian systems, Applied Mathematics Letters Volume 13, Issue 2 (2000) $63-70$.

[David \& Holm (1992)] D. David, D.D. Holm, Multiple Lie-Poisson Structures, Reductions, and Geometric Phases for the Maxwell-Bloch Travelling Wave Equations, J. Nonlinear Sci., Vol. 2 (1992) $241-262$.

[Fokas \& Fuchssteiner (1981] A.S. Fokas, B. Fuchssteiner, The Hierarchy of the Benjamin-Ono equations, Phys. Lett. 86 A (1981) 341-345.

[Fuchssteiner (1983)] B. Fuchssteiner, Mastersymmetries and higher order time-dependent symmetries and conserved densities of nonlinear evolution equations, Progr. Theor. Phys. 70 (1983) 1508-1522.

[Holm et al. (1991)] D.D. Holm, G. Kovacic, B. Sundaram, Chaotic laser-matter interaction, Physics Letters A 154 (1991) 346-352.

[Huang (2004)] D. Huang, Bi-Hamiltonian structure and homoclinic orbits of the MaxwellBloch equations with RWA, Chaos, Solitons and Fractals 22 (2004) 207-212.

[Lăzureanu et al. (2010)] C. Lăzureanu, T. Bînzar, F. Pater, On Periodic Solutions and Energy - Casimir Mapping for Maxwell-Bloch Equations (2010) 1245-1247, Annals of DAAAM for 2010 \& Proceedings of the 21st International DAAAM Symposium, ISBN 978-3-901509-73-5, ISSN 1726-9679, pp 0623, Editor B. Katalinic, Published by DAAAM International, Vienna, Austria 2010.

[Lăzureanu \& Bînzar (2012)] C. Lăzureanu, T. Bînzar, On the symmetries of a Rikitake type system, C. R. Acad. Sci. Paris, Ser. I 350 (2012) 529-533.

[Lăzureanu \& Bînzar (2013)] C. Lăzureanu, T. Bînzar, Symplectic realizations and symmetries of a Lotka-Volterra type system, Regular and Chaotic Dynamics, Vol. 18, No. 3 (2013) 203-213.

[Leach (1981)] P.G. Leach, A further note on the Henon-Heiles problem, J. Math. Phys. 22 (1981) 679-682.

[Libermann \& Marle (1987)] P. Libermann, C.-M. Marle, Symplectic Geometry and Analytical Mechanics, D. Reidel, Dordrecht, 1987.

[Marsden \& Raţiu (1999)] J. Marsden, T.S. Raţiu, Introduction to Mechanics and Symmetry, 2nd Ed. Text and Appl. Math. 17, Springer, Berlin, 1999.

[Noether (1918)] E. Noether, Invariante Variationsprobleme. Nachr. v. d. Ges. d. Wiss. zu Gottingen, Math. Phys. Kl (1918) 235-257; English translation, Transp. Th. Stat. Phys. 1 (1971) 186-207.

[Olver (1986)] P.J. Olver, Applications of Lie groups to Differential Equations, Springer Verlag, New York, 1986.

[Puta (1998)] M. Puta, Integrability and geometric prequantization of the Maxwell-Bloch equations, Bull. Sci. Math, Volume 122 (1998) 243-250.

[Puta (1996)] M. Puta, Three-dimensional real-valued Maxwell-Bloch equations with controls, Reports on Mathematical Physics, Volume 37, Issue 3 (1996) 337-348. 